\title{
Influência do uso de fluoxetina sobre o tempo de corrida em esteira ergométrica em ratos não treinados
}

CDD. 20.ed. 796.021

796.071
Douglas Colombi CUQUETTO*

José Geraldo MILL**

Fabian Tadeu do AMARAL ${ }^{* * *}$

\section{Resumo}

Trabalhos de diferentes autores constataram que, um aumento na concentração de serotonina (5-HT) no cérebro, durante exercício físico intenso e prolongado, está diretamente relacionado a um prejuízo na função do Sistema Nervoso Central (SNC), com correspondente desenvolvimento da fadiga e consequente diminuição do desempenho. No presente trabalho, 10 ratos Wistar foram submetidos a sessões de corrida até 0 esgotamento físico em esteira rolante. Os protocolos experimentais foram divididos em quatro fases: Fase 1 - avaliação do tempo médio de corrida dos animais até o esgotamento físico; Fase 2 - avaliação do tempo médio de corrida dos animais até o esgotamento físico, sob tratamento com fluoxetina; Fase 3 - "washout" de sete dias; Fase 4 - "washout" de 14 dias. Este estudo sugere que a administração crônica de fluoxetina aumenta substancialmente o tempo médio de corrida até 0 esgotamento físico em ratos submetidos a exercício em esteira rolante.

UnITERMos: Fluoxetina; Sistema nervoso; Exaustão.

\section{Introdução}

Pesquisas na área da fadiga durante exercício focaram, principalmente, a fadiga periférica, a qual envolve redução na habilidade do músculo em executar trabalho devido a prejuízos em algum local ao longo da cadeia de comando da transmissão neuromuscular às pontes cruzadas de actina e miosina (DAVIS, ALDERSON \& WeLSH, 2000). No entanto, o estimulo para a contração muscular é iniciado no cérebro, e por isso, a fadiga central pode ocorrer se alteraçóes dentro do Sistema Nervoso Central (SNC) diminuírem a capacidade de envio voluntário do estímulo para a junção neuromuscular (DAVIS \& BAILEY, 1997).

A fadiga é interpretada como um sinalizador de que a intensidade do exercício previamente tolerada deve ser reduzida. Em nível bioquímico podemos considerar a fadiga periférica como a perda da força e potência que ocorre independente da ação neuronal, pois nos músculos em atividade ocorrem diversas alteraçōes tais como: depleção de fosfocreatina, acúmulo de prótons e fosfato (HoOMANN \& STRUDER, 1996). Já na fadiga central, as baixas nas concentraçôes de glicose sanguínea devido à depleção do glicogênio

muscular e cerebral, o aumento da síntese de alguns neurotransmissores, bem como de seus precursores, são responsáveis por acarretar uma diminuição da ativação motoneural e impossibilidade de manter o esforço durante o exercício (Duarte, Dias \& Melo, 2008).

Newsholme e Blomstrand (1995) foram os primeiros a formularem a hipótese de que a serotonina, ou 5-hidroxitriptamina (5-HT), pudesse desempenhar um papel como mediador da fadiga central. Também foi questionado se o exercício prolongado poderia estimular importantes fatores que controlariam a síntese e turnover de 5-HT no cérebro, afetando assim, a performance do exercício.

O aumento da síntese de 5-HT no cérebro ocorre em resposta a um aumento na oferta de triptofano (TRP), fornecido pelo sangue, um aminoácido precursor de 5-HT. A maior parte do TRP circulante no plasma sanguíneo está ligado à albumina, $50 \mu \mathrm{M}(90 \%)$, embora também haja uma pequena porção, $5 \mu \mathrm{M}(10 \%)$, que circule na forma livre (TRP-l) e é transportado através da barreira hematoencefálica (Rossi \& TIRAPEGUI, 2004). Este transporte ocorre via receptor específico,
*Instituto Federal de Educação, Ciência e Tecnologia do Espírito Santo.

${ }^{* *}$ Centro de Ciências da Saúde, Universidade Federal do Espírito Santo.

${ }^{* * *}$ Centro de Educação Física e Desportos Universidade Federal do Espírito Santo. 
$A=$ albumina; TRP = triptofano; TRP-I = triptofano livre: $5-\mathrm{HT}=$ serotonina; $\mathrm{AG}=$ ácido graxo; BCAA = aminoácido de cadeia ramificada. que o TRP compartilha com outro grande aminoácido neutro, mais notadamente os aminoácidos de cadeia ramificada (BCAAs), tais como leucina, isoleucina e valina. Assim, a síntese de 5-HT no plasma aumenta quando existe um aumento na proporção da concentração de TRP-l para a concentração total de BCAAs no plasma, ou seja, quando TRP-l:BCAAs aumenta. Este aumento foi proposto para ocorrer durante o exercício prolongado por duas razões: primeiro, os BCAAs são captados do sangue e oxidados para fornecerem energia durante a contração do músculo esquelético e segundo, a concentração de ácidos graxos (AG) no plasma aumenta, causando um aumento paralelo de TRP-l, devido à substituição por AG nos sítios ativos do TRP na albumina. Tais eventos promoveriam diminuição da motivação, cansaço, letargia e menor coordenação motora, comprometendo a performance (CHAOULOFF, 1997; Rossi \& Tirapegui, 2000).

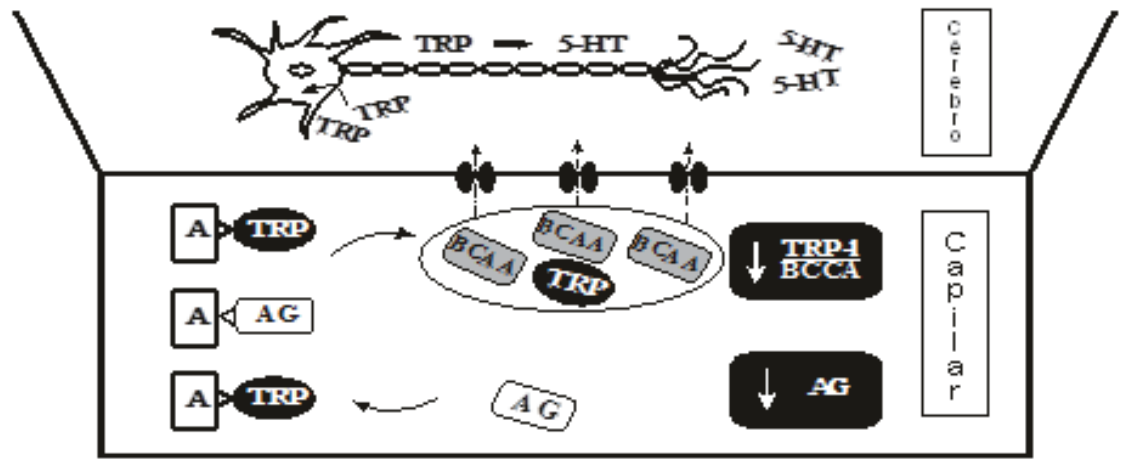

Repouso

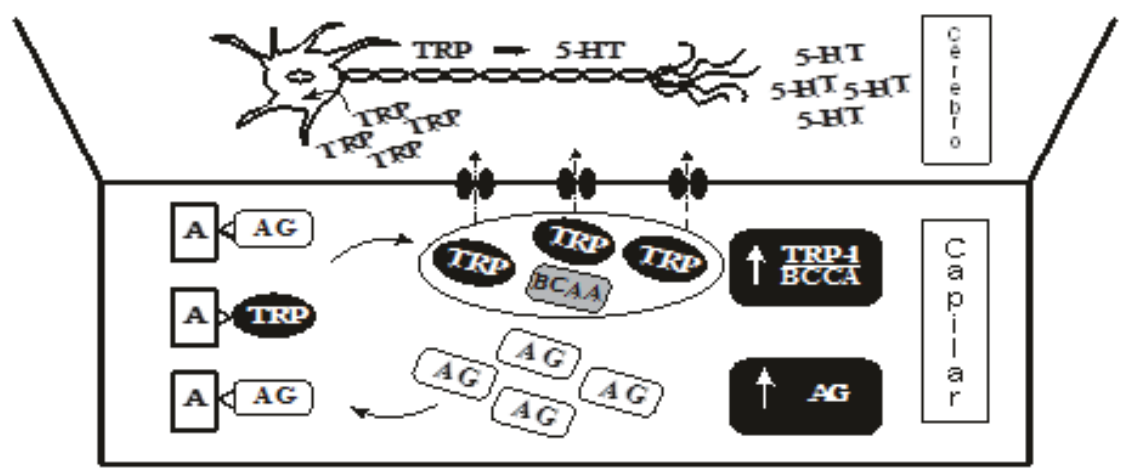

Exercício

FIGURA 1 - Esquema ilustrativo da Hipótese da Fadiga Central.

Pesquisas, tanto em ratos quanto em humanos, sustentam boa evidência sobre a atividade aumentada de 5-HT cerebral durante exercício prolongado e que essa resposta está associada à fadiga. Alguns pesquisadores estão apenas começando a explorar o possível mecanismo fisiológico que cerca essa resposta (DAVIS, Alderson \& Welsh, 2000).

Estudos mostram que a estimulação farmacológica de receptores 5-HT1c por clorophenylpiperazine $(m C P P)$, reduz o tempo de corrida até a exaustão e atenua a síntese de dopamina cerebral durante o exercício no núcleo "estriatum", mesencéfalo e hipocampo (BAILEY, DAVIS \& AHLBORN, 1992).
Fármacos agonistas e antagonistas 5-HT poderiam comprometer, também, a performance de "endurance" por afetar a termorregulação, agindo em receptores do subgrupo $5-\mathrm{HT}_{1 \mathrm{c}}$ e $5-\mathrm{HT}_{2 \mathrm{c}}$. A administração de 8- $\mathrm{OH}$ DPAT, um agonista $5-\mathrm{HT}_{1 \mathrm{c}}$, tem mostrado associação com efeito hipotérmico em ratos (HJORTH, 1985). Outro estudo, envolvendo pizotifen, um antagonista $5-\mathrm{HT}_{2 c}$, demonstrou hipertermia em sete indivíduos submetidos a 90 minutos de teste em cicloergômetro (STRACHAN, LEIPER \& MAUGHAN, 2005). Acredita-se que o efeito dessas drogas é mediado por associação direta com o hipotálamo.

A melhor relação causa e efeito entre o aumento cerebral de 5-HT e fadiga, foi demonstrada em 
uma série de experimentos envolvendo alterações farmacológicas cerebrais durante exercício em ratos. Bailey, Davis e Ahlborn (1993), propuseram que se o 5-HT pudesse ser artificialmente aumentado por meio da administração de droga agonista 5-HT (quipazine dimaleate $[Q D]$ ), a fadiga poderia ocorrer precocemente. Em contraste, se antagonistas 5-HT (LY 53.857 ou xylamidine tosylate [XT]) fossem administrados, a fadiga poderia ser retardada. Esse experimento demonstrou que o tempo de corrida para exaustão diminuiu após a administração de agonistas específicos 5-HT, mas aumentou após a administração de antagonistas 5-HT. Essas modificações no tempo de corrida para fadiga ocorreram apesar de não haver alteraçõos aparente na temperatura corporal, glicose sanguínea, glicogênio muscular e livre, ou variáveis hormonais.

A fluoxetina é uma droga inibidora da recaptação de serotonina no SNC, amplamente usada em

\section{Método}

Após aprovação, sobre aspectos éticos, por parte do Programa de Pós-Graduação em Ciências Fisiológicas da Universidade Federal do Espírito Santo, um grupo de 10 ratos Wistar, selecionados para corrida, com aproximadamente 60 dias de vida e pesando $200 \mathrm{~g}$, em média, foram submetidos a sessóes de corrida em esteira rolante ("Insight") com velocidade constante de $21 \mathrm{~m} / \mathrm{min}$ e $5^{\circ}$ de inclinação, conforme protocolo adotado por BaILEY, DaVIS e AhlBorn (1992). Essa esteira possui uma estrutura metálica com potencial elétrico localizada na parte posterior de cada baia. Os animais passaram por quatro sessôes de corrida (quatro dias consecutivos), com duração de cerca de 90 minutos, na esteira como processo de adaptação para os experimentos. $\mathrm{O}$ primeiro protocolo experimental (Fase 1) consistiu na avaliação do tempo médio de corrida dos animais até o esgotamento físico, divididos em três sessões semanais em dias alternados. No dia seguinte ao fim da Fase 1 (Fase 2) os animais iniciaram tratamento, durante sete dias, com fluoxetina $(10 \mathrm{mg} / \mathrm{kg} / \mathrm{dia})$ dissolvida em solução fisiológica $(\mathrm{NaCl} 0,9 \%)$ injetada intraperitonealmente (i.p.). Após esse período de tratamento, no qual os animais não correram, repetiu-se o mesmo protocolo da Fase 1 para avaliar tratamentos neurológicos como agente antidepressivo, por aumentar a neurotransmissão serotoninérgica cerebral (Wong, THRELKELD \& ROBERTSON, 1991); e utilizada também em programas para controle de peso corporal, sendo inibidora de apetite (HALFORD, HarRold, LaWTON \& Blundell, 2005). O aumento da atividade serotoninérgica poderia influenciar o tempo de exercício agindo em regiōes cerebrais como o córtex, hipotálamo, tálamo, substancia nigra e núcleo da rafe (Muck-Seler, Jevric-Causevic \& DiKSIC, 1996).

Os resultados obtidos nesse estudo poderão auxiliar na busca de um melhor esclarecimento sobre o papel do sistema serotoninérgico e sua relação com os processos de fadiga central e seu desenvolvimento.

Assim o objetivo deste estudo foi avaliar, por meio de pesquisa experimental, o efeito da administração de fluoxetina sobre o tempo de corrida de ratos sedentários em esteira rolante.

o tempo das sessões de corrida, porém sem interrupção do tratamento com a droga, totalizando 12 dias de tratamento com fluoxetina. Decorrido sete dias do encerramento da Fase 2, período esse em que foi suspendido o tratamento com a droga e as sessões de corrida, repetiu-se o protocolo da Fase 1, para mensuração do tempo até a exaustão em três sessões de corrida, para avaliar se a fluoxetina ainda promovia efeitos em nível central, ou se havia sido completamente metabolizada pelo organismo ("washout" de sete dias ou Fase 3). Posteriormente, foi dado mais sete dias (14 dias em relação à Fase 2), sem tratamento com a droga e sem realização de corrida, e novamente repetiu-se o protocolo para a avaliação do tempo até a fadiga ("washout" de 14 dias ou Fase 4). O estado de fadiga foi definido como o ponto no tempo durante o qual os animais desistiam da corrida, demonstrada por sujeição a estímulos elétricos longos ou consecutivos, num intervalo de aproximadamente um minuto.

Os resultados da pesquisa foram avaliados pelo teste t de "Student" para dados pareados. Neste caso, os dois valores originais, as duas médias obtidas antes e após tratamento, são provenientes do mesmo grupo de animais. Nível de significância adotado: 5\%. 


\section{Resultados}

O estudo teve duração de aproximadamente 40 dias e ao final da pesquisa os ratos tiveram ganho médio de $160 \mathrm{~g}$ de peso corporal, conforme mostrado na FIGURA 2.

Pela análise destes resultados, observa-se que a fluoxetina não demonstrou inibição de apetite, conforme trabalhos previamente mencionados.
O tempo de corrida durante a administração de fluoxetina aumentou cerca de 90 minutos, na média de tempo, comparado ao tempo pré-tratamento. Como houve diferença entre estes dois grupos, ou seja, manifestou-se algum efeito pelo tratamento, foi realizado "washout" de sete e 14 dias, períodos estes que comprovaram o efeito da fluoxetina, já que o tempo para esgotamento tendeu a voltar aos valores de repouso.
Diferença significante, $p<0,01$ (t Student).
Os dados estão representados em média \pm EPM;

Diferença significante em relação ao controle; ${ }^{*} p<0,05$ (t Student).

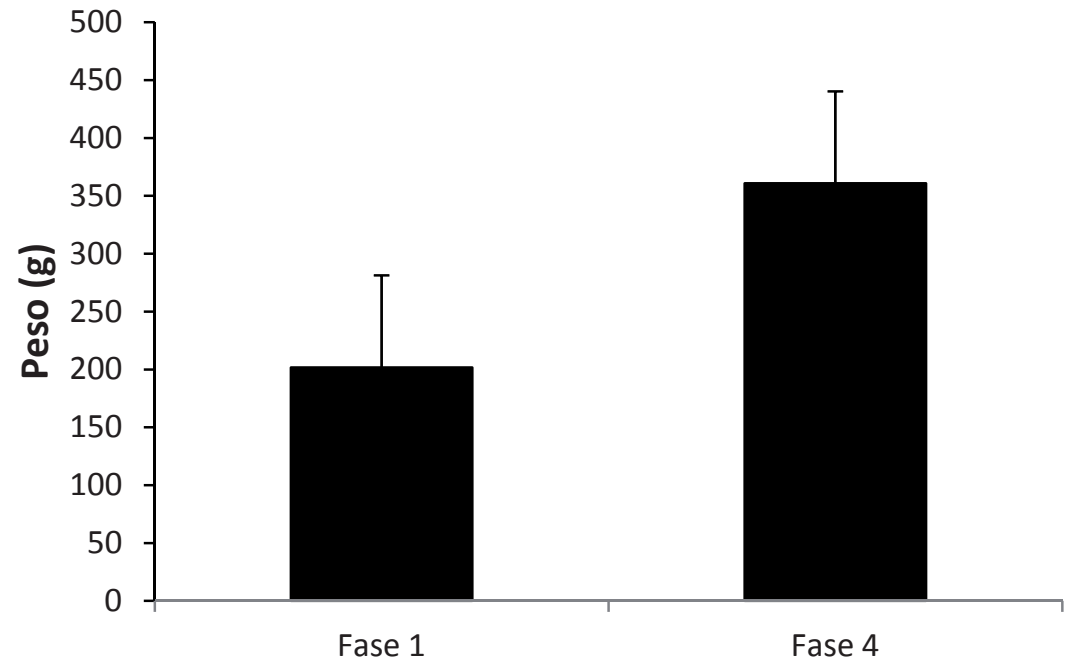

FIGURA 2 - Pesos dos animais antes da Fase 1 e após a Fase 4 (média e EPM).

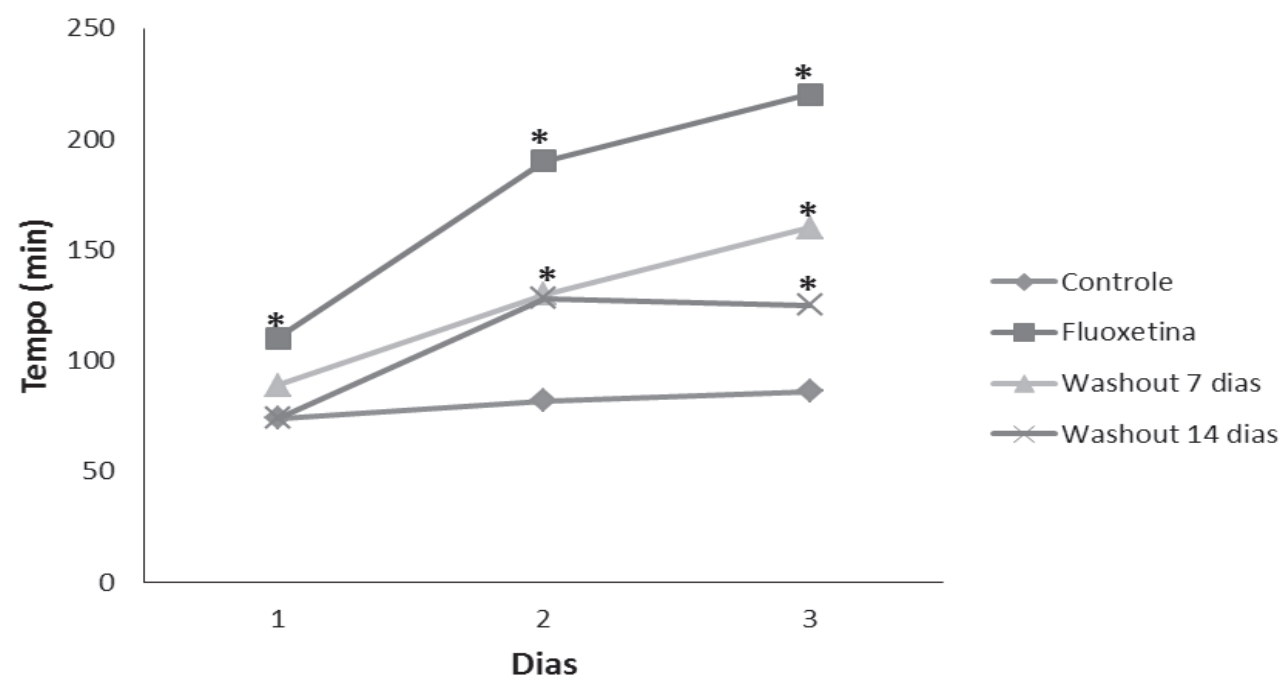

FIGURA 3 - Desempenho dos animais nas fases e nos dias em que foram realizados os testes. 


\title{
Discussão
}

Pouco se conhece sobre o mecanismo envolvendo o SNC e a fadiga durante o exercício. Pesquisas científicas têm sido amplamente limitadas nessa área devido, em grande parte, à falta de teorias viáveis que expliquem o modo como a fadiga ocorre, e à falta de boas metodologias para medir imediatamente e distinguir a fadiga central da fadiga periférica.

Estes resultados vão de encontro à "hipótese da fadiga central" descrita por NewSHOLMe e BlomsTRAND (1995) de que o aumento da atividade serotoninérgica no SNC comprometeria o desempenho durante exercícios prolongados; e contra alguns trabalhos que usaram a fluoxetina como possível agente para melhorar o desempenho de humanos em exercícios prolongados, onde não foram observados efeitos expressivos (Meeusen, Piacentrini, Van Den Eynde, Magnus \& De Meirleir, 2001; Wearden, Morris, Mullis, Strickland, Pearson, Appleby, Campbell \& Morris, 1998). No entanto, é uma das primeiras investigações que descreve um positivo efeito da administração de um agente 5 -HT agonista sobre a performance de "endurance" (tempo de corrida até exaustão) em ratos. Os mecanismos envolvidos no efeito de agonistas 5-HT sobre o tempo de exercício até a exaustão são incertos.

A hipótese provável para este aumento da performance da atividade física mediado pela fluoxetina, poderia ser devido à seu efeito antidepressivo por aumentar a disponibilidade de serotonina no SNC, proporcionando sensação de bem estar, o que aumentaria a motivação para a realização de atividades consideradas extenuantes.

Já nas fases de "washout", mesmo com a queda do tempo médio até o esgotamento físico, houve diferença significante em relação ao controle, que pode ser explicado pelo efeito promovido pelo treinamento, já que 14 e 21 dias ("washout" de sete e 14 dias respectivamente, comparados com o dia de início dos testes) de treinamento em corrida é suficiente para promover adaptações (DANTAS, 2003).

Uma das grandes dificuldades de se estudar a fluoxetina consiste no fato dessa droga ser um agonista não seletivo, ou seja, atua nos inúmeros receptores serotoninérgicos, os quais, estão envolvidos em diferentes funçôes.

Pela análise dos resultados desse estudo, conclui-se que a administração crônica (12 dias) de fluoxetina numa dosagem de $10 \mathrm{mg} / \mathrm{kg} / \mathrm{dia}$ (i.p.) aumenta pronunciadamente o tempo de corrida até o esgotamento físico em ratos submetidos a exercício em esteira rolante.

Contudo, há necessidade de mais pesquisas, envolvendo este tema para verificar o papel da fluoxetina e outros inibidores da recaptação de serotonina no SNC, como prováveis substâncias dopantes, bem como, elucidar o possível mecanismo responsável por esta resposta.

\begin{abstract}
Influence of using fluoxetine on run-time in treadmill in rats un trained

Works of different authors have been shown that an increase in the concentration of serotonin (5-HT) in the brain during intense and long-term physical exercise, is directly associated with a harm in the function of the Central Nervous System (CNS), with corresponding development of the fatigue and consequence decrease of the performance. In the present work, 10 rats Wistar were submitted to runsessions until the exhaustion in treadmill. The experimental protocols were splited in 4 phases: Phase 1: evaluation of the range run-time of the animals until the physical exhaustion. Phase 2: evaluation of the range run-time of the animals until the physical exhaustion on treatment with fluoxetine. Phase 3 : washout seven days. Phase 4 washout 14 days. The present study suggest that chronic administration of fluoxetine increase substantially the run-time until the physical exhaustion in rats submitted to exercises in treadmill.
\end{abstract}

UnIterms: Fluoxetin; Nervous system; Exhaustion. 


\section{Resumen}

Efecto de la fluoxetina sobre el tiempo de ejecución en una cinta en ratas no entrenadas

Las obras de diferentes autores encontraron que un aumento en la concentración de la serotonina (5-HT) en el cerebro durante el ejercicio intenso y prolongado está directamente relacionado con deterioro de la función del Sistema Nervioso Central (SNC), con el correspondiente desarrollo de la fatiga y su consecuente reducción en el rendimiento. En este estudio, 10 ratas Wistar fueron sometidas a la ejecución de sesiones en una cinta hasta el agotamiento físico. Los protocolos experimentales fueron divididos en cuatro fases: Fase 1 - Evaluación de la carrera media de los animales al agotamiento físico, la Fase 2 - Evaluación de la carrera media de los animales al agotamiento físico, están en tratamiento con fluoxetina, la Fase 3 - "lavado "de siete días; Fase 4 -" lavado "de 14 días. Este estudio sugiere que la administración crónica de fluoxetina incrementa sustancialmente la tarifa media del agotamiento físico en ratas sometidas a ejercicio en una caminadora.

Palabras clave: La fluoxetina; El sistema nervioso; Agotamiento.

\section{Referências}

BAILEY, S.P; DAVIS, J.M.; AHLBORN, E.N. Effect of increased brain serotonergic (5-HT1c) activity on endurance performance in the rat. Acta Physiologica Scandinavica, San Francisco, v.145, p.76-77, 1992.

Serotonergic agonists and antagonists affect endurance performance in the rat. International Journal of Sports

Medicine, New York, v.14, p.330-3, 1993.

CHAOULOFF, F. Effects of acute physical exercise on central serotonergic systems. Medicine and Science in Sports and Exercise, Madison, v.29, n.1, p.58-62, 1997.

DANTAS, E.H. A prática da preparação física. Rio de Janeiro: Shape, 2003.

DAVIS, J.M.; ALDERSON, N.L.; WELSH R. Serotonin and central nervous system fatigue: nutritional considerations. American Journal of Clinical Nutrition, Bethesda, v.72, p. 3-8, 2000. Supplement 57.

DAVIS, J.M.; BAILEY, S.P. Possible mechanisms of central nervous system fatigue during exercise. Medicine and Science in Sports and Exercise, Madison, v.29, n.1, p.45-57, 1997.

DUARTE, V.L.; DIAS, D.S.; MELO, H.C.S. Molecular mechanisms of fatigue. Brazilian Journal of Biomotricity, Rio de Janeiro, v.2, n.1, p.3-38, 2008.

HALFORD, J.C.; HARROLD, J.A.; LAWTON, C.L.; BLUNDELL, J.E. Serotonin (5-HT) drugs: effects on appetite expression and use for the treatment of obesity. Current Drug Targets, Notre Dame, v.6, n.2, p.201-13, 2005.

HJORTH, S. Hypothermia in the heat induced by the potent serotonergic agent 8-OH-DPAT. Journal of Neural Transmission, New York, v.61, n.1-2, p.671-7, 1985.

HOOMANN, W.; STRUDER, H.K. Exercise, physical activity, nutrition, and the brain. Nutrition Reviews, Washington, v.54, p.37-43, 1996.

MEEUSEN, R.; PIACENTRINI, M.F.; VAN DEN EYNDE, S.; MAGNUS, L; DE MEIRLEIR, K. Exercise performance is not influenced by a 5-HT reuptake inhibitor. Sports Medicine, Auckland, v.22, n.3, p.29-36, 2001.

MUCK-SELLER, D.; JEVRIC-CAUSEVIC, A.; DIKSIC, M. Influence of fluoxetine on regional serotonin synthesis in the rat brain. Journal of Neurochemistry, Washington, v.67, n.2, p.434-42, 1996.

NEWSHOLME, E.A.; BLOMSTRAND, E. Tryptophan, 5-hydroxytryptamine and a possible explanation for central fatigue. Advances in Experimental Medicine and Biology, Heidelberg, v.384, p.315-20, 1995.

ROSSI, L.; TIRAPEGUI, J.O. Aminoácidos: bases atuais para sua suplementação na atividade física. Revista Brasileira de Ciências Farmacêuticas, São Paulo, v.36, n.1, p.37-51, 2000.

Implicaçôes do sistema serotoninérgico no exercício físico. Arquivos Brasileiros de Endocrinologia e Metabologia, São Paulo, v.48, n.2, p.227-33, 2004.

STRACHAN, A.T.; LEIPER, J.B.; MAUGHAN, R.J. Serotonin2c receptor blockade and thermoregulation during exercise in the heat. Medicine and Science in Sports and Exercise, Madison, v.37, n.3, p.389-394, 2005. 
WEARDEN, A.J.; MORRIS, R.K.; MULLIS, R.; STRICKLAND, P.L.; PEARSON, D.J.; APPLEBY, L.; CAMPBELL, I.T.; MORRIS, J.A. Randomised, double-blind, placebo-controlled treatment trial of fluoxetine and graded exercise for fatigue syndrome. British Journal of Psychiatry, London, v.172, p.485-90, 1998.

WONG, D.T., THRELKELD, P.G.; ROBERTSON, D.W. Affinities of fluoxetine its enantiomers and other inhibitors of serotonin uptake for subtypes of serotonin receptors. Neuropsychopharmacology, New York, v.5, n.1, p.43-7, 1991.

Douglas Colombi Cuquetto

R. Nova Conceição, 246 29830-00o - Nova Venécia - ES - BRASIL e-mail: douglascolombi@hotmail.com
Recebido para publicação: 13/07/2009

Revisado: 10/05/2012

Aceito: 24/05/2012 